\title{
Spin-orbit torques in two-dimensional Rashba ferromagnets
}

\author{
A. Qaiumzadeh, ${ }^{1,2}$ R. A. Duine, ${ }^{3}$ and M. Titov ${ }^{1}$ \\ ${ }^{1}$ Institute for Molecules and Materials, Radboud University, 6525 AJ Nijmegen, The Netherlands \\ ${ }^{2}$ Department of Physics, Institute for Advanced Studies in Basic Sciences (IASBS), Zanjan 45137-66731, Iran \\ ${ }^{3}$ Institute for Theoretical Physics and Centre for Extreme Matter and Emergent Phenomena, Utrecht University, \\ 3584 CE Utrecht, The Netherlands
}

(Received 23 March 2015; published 6 July 2015)

\begin{abstract}
Magnetization dynamics in single-domain ferromagnets can be triggered by a charge current if the spin-orbit coupling is sufficiently strong. We apply functional Keldysh theory to investigate spin-orbit torques in metallic two-dimensional Rashba ferromagnets in the presence of spin-dependent disorders. A reactive, antidamping-like spin-orbit torque as well as a dissipative, field-like torque is calculated microscopically, to leading order in the spin-orbit interaction strength. By calculating the first vertex correction we show that the intrinsic antidampinglike torque vanishes unless the scattering rates are spin dependent.
\end{abstract}

DOI: 10.1103/PhysRevB.92.014402

PACS number(s): 75.76.+j, 72.15.Gd, 75.60.Jk, 75.70.Tj

\section{INTRODUCTION}

Spin-orbitronics [1,2] has attracted a lot of attention recently as a new subfield of spintronics [3,4] in which the relativistic spin-orbit interaction (SOI) plays a central role. Spin-orbitronics includes the generation and detection of spin-polarized currents through the spin Hall effect $[5,6]$, the induction of nonequilibrium spin accumulations in nonmagnetic materials through the Edelstein effect [7,8], the triggering of magnetization dynamics in single magnetic systems through spin-orbit torques (SOTs) [9-11], and magnonic charge pumping by means of inverse SOTs [12]. Spin-orbitronics is believed to ultimately enablethe faster and more efficient ways of magnetization switching needed for high-density data storage and information processing, thereby providing novel solutions to address the essential challenges of spintronics. In this paper we investigate the microscopic origin of SOTs in a two-dimensional (2D) metallic ferromagnet with spin-orbit coupling.

The magnetization dynamics in ferromagnets is governed by the seminal Landau-Lifshitz-Gilbert (LLG) equation [13$15]$,

$$
\frac{\partial \boldsymbol{m}}{\partial t}=-\gamma \boldsymbol{m} \times \boldsymbol{H}_{\mathrm{eff}}+\alpha_{G} \boldsymbol{m} \times \frac{\partial \boldsymbol{m}}{\partial t}+\boldsymbol{T},
$$

where $\boldsymbol{m}$ is a unit vector along the magnetization direction $|\boldsymbol{m}|=1, \gamma$ is the gyromagnetic ratio, $\alpha_{G}$ is the Gilbert damping constant, and $\boldsymbol{H}_{\mathrm{eff}}$ is an effective field which includes the effects of the external magnetic field, exchange interactions, and dipole and anisotropy fields. The first term on the right-hand side of Eq. (1) describes the precession of the magnetization vector $\boldsymbol{m}$ around the effective field, while the second term describes the relaxation of magnetization to its equilibrium orientation. Furthermore, $\boldsymbol{T}$ is a sum of different magnetization torques not contained in the effective field or damping.

The spin-polarized current-induced magnetization dynamics in magnetic materials arises as a result of spin transfer torque (STT) [13-15]. It is well known that STT may induce magnetization dynamics in spin-valve structures and that the exchange interaction between the spin-polarized current and local spins leads, e.g., to domain-wall motion. In uniformly magnetized single-domain systems the transfer of spin angular momentum from the spin-current density $\boldsymbol{j}_{s}$ to a local magnetization is modeled by two different STT terms: (i) an antidamping-like (ADL) or Slonczewski in-plane torque, $\boldsymbol{T} \propto$ $\boldsymbol{m} \times \boldsymbol{m} \times \boldsymbol{j}_{s}$; and (ii) an out-of-plane field-like (FL) torque, $\boldsymbol{T} \propto \boldsymbol{m} \times \boldsymbol{j}_{s}$, which is typically negligible in conventional metallic spin valves. On the other hand, in ferromagnets with magnetic domains, in which spin textures such as domain walls are necessarily present, the STT also includes reactive, $\boldsymbol{T} \propto\left(\boldsymbol{j}_{s} \cdot \nabla\right) \boldsymbol{m}$, and dissipative, $\boldsymbol{T} \propto \boldsymbol{m} \times\left(\boldsymbol{j}_{s} \cdot \nabla\right) \boldsymbol{m}$, torques [13-15].

Recently, it was demonstrated both theoretically and experimentally that the current-induced nonequilibrium spin polarization [7,8] in (anti-)ferromagnets with inversion asymmetry may exert a so-called SOT on localized spins and, consequently, may lead to a nontrivial magnetization dynamics [16-32]. Unlike STT, the SOT phenomenon does not require an injection of spin current or the presence of spatial inhomogeneities in the magnetization. The magnetization switching due to SOTs may be achieved with current pulses as short as $\sim 180$ ps, while the critical charge current density can be as low as $\sim 10^{7} \mathrm{~A} \mathrm{~cm}^{-2}$ [29].

Quite generally Rashba SOTs can be classified as either ADL or FL torques [33]. The first theoretical and experimental studies of SOT have demonstrated that the ADL SOT is proportional to the disorder strength and can always be regarded as a small correction to the FL SOT [16-23]. On the other hand, in some recent experiments the opposite statement is made: torques with ADL symmetry are more likely to be the main source of the observed magnetization behavior [26-28,34-37]. These experiments are performed with ferromagnetic metals grown on top of a heavy metal with strong SOI and may, in principle, be explained by the spin Hall effect, which induces a spin-polarized current. This spin current, in turn, exerts a torque on the magnetic layer via the STT mechanism $[35,36,38]$ so that the ADL symmetry term plays the major role in the effect as discussed above.

It is, however, a serious experimental challenge to distinguish between SOT and spin Hall STT in bilayers, since both torques have the same symmetry [35,36]. Very recently Kurebayashi et al. [39] conducted an experiment on the bulk of strained GaMnAs, which has an intrinsic crystalline asymmetry. In these experiments, the contribution 
of a possible spin-Hall-effect STT was completely eliminated, while sizable ADL torques were nevertheless detected. This provides a strong argument in favor of the ADL-SOT nature of the observed torque. The authors of Ref. [39] attribute this torque to an intrinsic Berry curvature and estimate a scattering-independent, i.e., intrinsic, ADL SOT [39-41]. This intrinsic ADL SOT has also been reported by van der Bijl and Duine [33].

In this paper we calculate both FL and ADL SOTs in a 2D Rashba ferromagnetic metal microscopically by using a functional Keldysh theory approach [42]. By calculating the first vertex correction we show that the intrinsic ADL SOT vanishes unless the impurity scattering is spin dependent.

The rest of the paper is organized as follows. Section II introduces the model and method. In Sec. III we calculate SOTs with and without vertex corrections. We conclude our work in Sec. IV.

\section{MODEL HAMILTONIAN AND METHOD}

We start with the 2D mean-field Hamiltonian $(\hbar=c=1)$,

$$
\mathcal{H}\left[\psi^{\dagger}, \psi\right]=\int d^{2} \boldsymbol{r} \psi_{\boldsymbol{r}, t}^{\dagger}\left[H_{0}+V_{\mathrm{imp}}+\hat{\boldsymbol{j}} \cdot \boldsymbol{A}_{t}\right] \psi_{\boldsymbol{r}, t},
$$

where $\boldsymbol{\psi}^{\dagger}=\left(\boldsymbol{\psi}_{\uparrow}^{*}, \boldsymbol{\psi}_{\downarrow}^{*}\right)$ is the Grassman coherent-state spinor. Here, $H_{0}$ is the $2 \mathrm{D}$ conducting ferromagnet Hamiltonian density in the presence of Rashba SOI [43],

$$
H_{0}=\frac{\boldsymbol{p}^{2}}{2 m_{e}}+\alpha_{R}(\boldsymbol{\sigma} \times \hat{z}) \cdot \boldsymbol{p}-\frac{1}{2} \Delta \boldsymbol{\sigma} \cdot \boldsymbol{n}_{\boldsymbol{r}, t}-\frac{1}{2} \Delta_{B} \sigma_{z},
$$

where $\boldsymbol{p}$ is the 2D momentum operator, $\alpha_{R}$ is the strength of the SOI; $\Delta$ and $\Delta_{B}$ are the exchange energy and the Zeeman splitting due to an external field in the $z$ direction, respectively; $\boldsymbol{n}_{\mathbf{r}, t}$ is an arbitrary unit vector that determines the quantization axis; and $\sigma$ is the three-dimensional vector of Pauli matrices.

The vector potential $\boldsymbol{A}_{t}=\boldsymbol{E} e^{-i \Omega t} / i \Omega$ is included in Eq. (2) to model a dc electric field in the limit $\Omega \rightarrow 0$. It is coupled to the current density operator, which is given by $\hat{\boldsymbol{j}}=\left(i e / 2 m_{e}\right)(\overleftarrow{\nabla}-\vec{\nabla})-e \alpha_{R} \sigma \times \hat{z}$, where $e$ is the electron charge and $m_{e}$ is the electron effective mass. Finally, the impurity potential $V_{\text {imp }}$ is of the form

$$
V_{\mathrm{imp}}(\boldsymbol{r})=\left(\begin{array}{cc}
V_{\uparrow} & 0 \\
0 & V_{\downarrow}
\end{array}\right) \sum_{i} \delta\left(\mathbf{r}-\mathbf{R}_{i}\right),
$$

where $V_{\uparrow(\downarrow)}$ is the strength of spin-up (down) disorder, and the index $i$ labels the impurity centers $\mathbf{R}_{i}$. More specifically, we restrict ourselves to the Gaussian limit of the disorder potential.

The impurity-averaged retarded Green's function in the Born approximation is given by [44-46]

$$
G_{\boldsymbol{k}, \varepsilon}^{+}=\left(g_{\downarrow}^{-1} \sigma^{\uparrow}+g_{\uparrow}^{-1} \sigma^{\downarrow}+\alpha_{R}\left(\sigma_{y} k_{x}-\sigma_{x} k_{y}\right)\right)^{-1},
$$

where $g_{s}^{-1}=\varepsilon-\varepsilon_{k}+s M+i \gamma_{s}$, for $s=\uparrow(+)$ or $s=\downarrow(-)$, $\sigma^{s}=\left(\sigma_{0}+s \sigma_{z}\right) / 2, \boldsymbol{k}$ and $\varepsilon$ are the wave vector and energy, respectively, $\varepsilon_{k}=k^{2} / 2 m_{e}$, and $M=\left(\Delta+\Delta_{B}\right) / 2$. We have also introduced the spin-dependent scattering rate $\gamma_{s}=\pi \nu n_{\mathrm{imp}} V_{s}^{2}$, where $\nu_{0}=m_{e} / 2 \pi$ is the density of states per spin for a $2 \mathrm{D}$ electron gas, and $n_{\text {imp }}$ denotes the impurity concentration. Here we have assumed that both spin-orbit split bands are occupied, i.e., the Fermi energy is larger than the magnetization splitting, $\varepsilon_{F}>M$.

Following Ref. [42] we minimize the effective action on the Keldysh contour with respect to quantum fluctuations of $\boldsymbol{n}$. This procedure gives us directly the LLG equation, which contains torque terms in linear response with respect to the external field $\boldsymbol{E}$. The effective action is given by $S=\int_{\mathcal{C}^{K}} d t L_{F}(t)$, where $\mathcal{C}^{K}$ stands for the Keldysh contour and $L_{F}(t)=\int d^{2} \boldsymbol{r}\left(\hat{\boldsymbol{\psi}}_{\boldsymbol{r}, t}^{\dagger} i \frac{\partial}{\partial t} \hat{\boldsymbol{\psi}}_{\boldsymbol{r}, t}-\mathcal{H}\right)$ is the mean-field Lagrangian.

We further assume that we are dealing with a ferromagnetic metal which is uniformly magnetized in the $z$ direction. Thus, we can approximate the vector $\boldsymbol{n}$ as

$$
\boldsymbol{n}_{\boldsymbol{r}, t} \simeq\left(\begin{array}{c}
\delta n_{\boldsymbol{r}, t}^{x} \\
\delta n_{\boldsymbol{r}, t}^{y} \\
1-\frac{1}{2}\left(\delta n_{\boldsymbol{r}, t}^{x}\right)^{2}-\frac{1}{2}\left(\delta n_{\boldsymbol{r}, t}^{y}\right)^{2}
\end{array}\right) .
$$

In order to derive the LLG equation with torque terms it is sufficient to expand the effective action up to second order in $\delta \boldsymbol{n}$ and up to first order in the vector potential: $S_{\text {eff }}=S_{\text {SOT }}[\mathcal{O}(\delta \mathbf{n}), \mathbf{A}]+S_{\text {rest }}\left[\mathcal{O}\left(\delta \mathbf{n}^{2}\right), \mathbf{A}=0\right]$. A straightforward calculation gives

$$
S_{\mathrm{SOT}}=\int_{\mathcal{C}^{K}} d t \int_{\mathcal{C}^{K}} d t^{\prime} \int d^{2} \boldsymbol{r} \int d^{2} \boldsymbol{r}^{\prime} \chi_{a ; \boldsymbol{r}-\boldsymbol{r}^{\prime} ; t, t^{\prime}} \delta n_{\boldsymbol{r}^{\prime}, t^{\prime}}^{a},
$$

where $\chi_{a}(a=\{x, y\})$ is the response function,

$$
\chi_{a ; \boldsymbol{r}-\boldsymbol{r}^{\prime} ; t, t^{\prime}}=\frac{i \Delta}{4}\left\langle\boldsymbol{j}_{\boldsymbol{r}, t} \psi_{\boldsymbol{r}^{\prime}, t^{\prime}}^{\dagger} \sigma_{a} \psi_{\boldsymbol{r}^{\prime}, t^{\prime}}\right\rangle \cdot \boldsymbol{A}_{t},
$$

and $\boldsymbol{j}=\psi^{\dagger} \hat{\boldsymbol{j}} \psi$ is the charge current density. Note that in the absence of SOI the term $S_{\text {SOT }}$ is 0 and only second-order terms, $S_{\text {rest }}$ [42], remain. The field $\delta \boldsymbol{n}$ can be split into the physical magnetization field $\delta \mathbf{m}$ and a quantum fluctuation field $\boldsymbol{\xi}$ as $\delta n_{\boldsymbol{r}, t_{ \pm}}^{a}=\delta m_{\boldsymbol{r}, t}^{a} \pm \xi_{\boldsymbol{r}, t}^{a} / 2$, where a plus sign corresponds to the upper and a minus sign to the lower branch of the Keldysh contour. At first order with respect to the quantum component we obtain

$$
S_{\mathrm{SOT}}=\int d t \int d t^{\prime} \int d^{2} \boldsymbol{r}^{\prime} \int d^{2} \boldsymbol{r} \chi_{a ; \mathbf{r}-\mathbf{r}^{\prime} ; t, t^{\prime}}^{-} \xi_{\mathbf{r}^{\prime}, t^{\prime}}^{a},
$$

where $\chi^{-}$is the advanced component of the correlator, and the sum over repeated indices $a$ is assumed. The LLG equation is, then, derived by minimizing the effective action with respect to quantum fluctuations, $\delta S_{\text {eff }} / \delta \xi=0$. Thus, the transverse components of the LLG equation in the Fourier space are given by

$$
\mathcal{F}\left[\frac{\delta S_{\text {rest }}}{\delta \xi_{a}}\right]_{\mathbf{q}=0, \varepsilon}+\chi_{a ; \mathbf{q}=0, \varepsilon=0}^{-}=0,
$$

where $\mathcal{F}[\ldots]$ represents the Fourier transformation operator. The functional derivative in Eq. (10) gives the precession and Gilbert damping terms of the LLG equation [42], while the second term describes the SOT. The dependence of Gilbert damping on the SOI is second order in $\alpha_{R}$ [33], and we focus below on SOT which is of first order in $\alpha_{R}$. The appearance of the zero-momentum response function $\chi_{a ; \mathbf{q}=0, \varepsilon=0}$ in the LLG equation shows that the SOT is finite even for spatially uniform magnetization, in contrast to the (non-)adiabatic STT, which is of first order in the gradient of magnetization. 
(a)

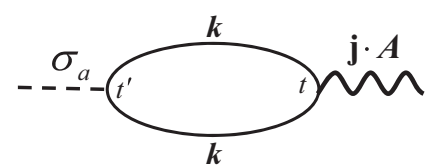

(b)

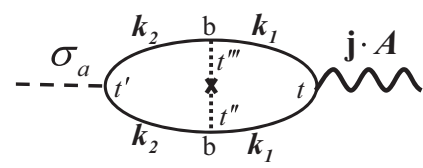

FIG. 1. Feynman diagrams related to the spin-torque response function, Eq. (8): (a) undressed response function and (b) first vertex correction. The solid line corresponds to an electron propagator in the Born approximation; the wiggly line, to the coupling to the vector potential and current; and the dashed line, a spin fluctuation. The vertical dotted line with an $\mathbf{x}$ describes the averaging over impurity positions.

\section{CALCULATION OF SOTs}

In what follows we evaluate the spin-torque response function of Eq. (8), shown diagrammatically in Fig. 1, to derive the SOT in the ballistic limit $\gamma_{s} \ll k_{B} T$, where $k_{B} T$ is the thermal energy. We calculate first the bare (undressed) part of the response function, $\chi^{(0)}$, depicted in Fig. 1(a). The final result for spin torque is, then, obtained by adding the first vertex correction, $\chi^{(1)}$, depicted in Fig. 1(b). Throughout the calculation we assume that $\gamma_{s} \ll k_{B} T \ll \alpha_{R} k_{F} \ll M$, where $k_{F}$ is the Fermi wave vector. The condition $\alpha_{R} k_{F} \ll M$ is normally fulfilled in the metallic ferromagnets of interest. Whether or not the condition $\gamma_{s} \ll k_{B} T \ll \alpha_{R} k_{F}$ is fulfilled depends strongly on the sample quality. The analysis of spin torques in the diffusive regime $k_{B} T \ll \gamma_{s}$ will require calculation of the full vertex correction and will be done elsewhere.

\section{A. Undressed response function}

The spin-torque response function of Eq. (8) without vertex corrections is given by

$$
\chi_{a ; t, t^{\prime}}^{(0)}=\frac{e \Delta}{4 i} \int \frac{d^{2} \boldsymbol{k}}{(2 \pi)^{2}} \operatorname{Tr}\left[\boldsymbol{v}_{\boldsymbol{k}} \check{G}_{\boldsymbol{k} ; t, t^{\prime}} \sigma_{a} \check{G}_{\boldsymbol{k} ; t^{\prime}, t}\right] \cdot \mathbf{A}_{t},
$$

where $\boldsymbol{v}_{\boldsymbol{k}}=\boldsymbol{k} / m_{e}-\alpha_{R} \boldsymbol{\sigma} \times \hat{z}$ is the velocity vector, and $\check{G}$ is the Green's function on the Keldysh contour. From Eq. (11) we find retarded and advanced components of the response function in the limit of zero frequency and momentum as

$$
\begin{aligned}
\chi_{a}^{(0) \pm}= & \frac{e \Delta}{4 i} \lim _{\Omega \rightarrow 0} \int \frac{d^{2} \boldsymbol{k}}{(2 \pi)^{2}} \iint d \omega d \omega^{\prime} \frac{f_{\omega^{\prime}}-f_{\omega}}{\Omega+\omega^{\prime}-\omega \pm i 0} \\
& \times \frac{1}{\Omega} \operatorname{Tr}\left[\left(\boldsymbol{v}_{\boldsymbol{k}} \cdot \boldsymbol{E}\right) \mathcal{A}_{\boldsymbol{k}, \omega} \sigma_{a} \mathcal{A}_{\boldsymbol{k}, \omega^{\prime}}\right],
\end{aligned}
$$

where $\mathcal{A}_{\boldsymbol{k}, \omega}=i\left(G_{\boldsymbol{k}, \omega}^{+}-G_{\boldsymbol{k}, \omega}^{-}\right) / 2 \pi$ is the spectral function and $f_{\omega}=\left[e^{\left(\omega-\epsilon_{F}\right) / k_{B} T}+1\right]^{-1}$ stands for the Fermi distribution function.

In the limit of weak disorder, we can decompose the response function into two parts: the intrinsic part $\chi_{\text {in }}$, which turns out not to depend on the scattering rate and describes interband transitions, and the extrinsic part $\chi_{\mathrm{ex}}$, which essentially depends on disorder and corresponds to intraband contributions. The intrinsic part corresponds to the principal value integration in Eq. (12), while the extrinsic part is given by the corresponding $\delta$-function contribution. To leading order in $\alpha_{R}$ we find

$$
\begin{aligned}
& \chi_{\mathrm{in}, \mathrm{a}}^{(0)-}=\frac{e \alpha_{R} \Delta}{8 M} v_{0} E_{a}, \\
& \chi_{\mathrm{ex}, \mathrm{a}}^{(0)-}=\frac{e \alpha_{R} \Delta}{8 M} v_{0}\left[\frac{\varepsilon_{F}-M}{\gamma_{\downarrow}}-\frac{\varepsilon_{F}+M}{\gamma_{\uparrow}}\right](\hat{z} \times \boldsymbol{E})_{a} .
\end{aligned}
$$

The corresponding expressions for SOTs are the ADL, $\mathbf{T}^{\mathrm{ADL}}$, and FL, $\mathbf{T}^{\mathrm{FL}}$, contributions, which do not take into account vertex corrections:

$$
\begin{aligned}
\boldsymbol{T}_{\mathrm{ADL}}^{(0)} & =-2 e \alpha_{R} v_{0} \boldsymbol{m} \times \boldsymbol{m} \times(\hat{z} \times \boldsymbol{E}), \\
\boldsymbol{T}_{\mathrm{FL}}^{(0)} & =-\frac{e \alpha_{R} \Delta v_{0}}{M}\left[\frac{\varepsilon_{F}+M}{\gamma_{\uparrow}}-\frac{\varepsilon_{F}-M}{\gamma_{\downarrow}}\right] \boldsymbol{m} \times(\hat{z} \times \boldsymbol{E}) .
\end{aligned}
$$

Hence, we find that the ADL SOT in the absence of vertex corrections has an intrinsic origin, i.e., is disorder independent.

\section{B. Vertex correction}

Let us now turn to the calculation of the first vertex correction to the spin-torque response function depicted in Fig. 1(b). For the corresponding response function on the Keldysh contour we find

$$
\begin{aligned}
\chi_{a ; t, t^{\prime}}^{(1)}= & \frac{e \Delta}{4 i} \int \frac{d \boldsymbol{k}_{1}}{(2 \pi)^{2}} \int \frac{d \boldsymbol{k}_{2}}{(2 \pi)^{2}} \int_{c^{K}} d t_{1} \int_{c^{K}} d t_{2} \operatorname{Tr}\left[\mathbf{A}_{t} \cdot \boldsymbol{v}_{\boldsymbol{k}_{1}}\right. \\
& \left.\times \check{G}_{\boldsymbol{k}_{1} ; t, t_{1}}\left\langle V_{\mathrm{imp}} \check{G}_{\boldsymbol{k}_{2} ; t_{1}, t^{\prime}} \sigma_{a} \check{G}_{\boldsymbol{k}_{2} ; t^{\prime}, t_{2}} V_{\mathrm{imp}}\right\rangle \check{G}_{\mathbf{k}_{1} ; t_{2}, t}\right] .
\end{aligned}
$$

The advanced component of $\chi^{(1)}$ at zero energy and momentum is, then, given by

$$
\begin{aligned}
\chi_{a}^{(1)-}= & \frac{e \Delta}{4 i} \eta_{b} \int \frac{d^{2} \boldsymbol{k}_{1}}{(2 \pi)^{2}} \int \frac{d^{2} \boldsymbol{k}_{2}}{(2 \pi)^{2}} \iint d \omega d \omega^{\prime} \frac{f_{\omega^{\prime}}-f_{\omega}}{\Omega+\omega-\omega^{\prime}-i 0} \\
& \times \frac{1}{\Omega} \operatorname{Tr}\left[\boldsymbol { E } \cdot \boldsymbol { v } _ { \mathbf { k } _ { 1 } } \left(G_{\boldsymbol{k}_{1}, \omega}^{+} \sigma_{b} \mathcal{A}_{\boldsymbol{k}_{2}, \omega} \sigma_{a} G_{\boldsymbol{k}_{2}, \omega^{\prime}}^{+} \sigma_{b} \mathcal{A}_{\boldsymbol{k}_{1}, \omega^{\prime}}\right.\right. \\
& +G_{\boldsymbol{k}_{1}, \omega}^{+} \sigma_{b} \mathcal{A}_{\boldsymbol{k}_{2}, \omega} \sigma_{a} \mathcal{A}_{\boldsymbol{k}_{2}, \omega^{\prime}} \sigma_{b} G_{\boldsymbol{k}_{1}, \omega^{\prime}}^{-} \\
& +\mathcal{A}_{\boldsymbol{k}_{1}, \omega} \sigma_{b} G_{\boldsymbol{k}_{2}, \omega}^{-} \sigma_{a} G_{\boldsymbol{k}_{2}, \omega^{\prime}}^{+} \sigma_{b} \mathcal{A}_{\boldsymbol{k}_{1}, \omega^{\prime}} \\
& \left.\left.+\mathcal{A}_{\boldsymbol{k}_{1}, \omega} \sigma_{b} G_{\boldsymbol{k}_{2}, \omega}^{-} \sigma_{a} \mathcal{A}_{\boldsymbol{k}_{2}, \omega^{\prime}} \sigma_{b} G_{\boldsymbol{k}_{1}, \omega^{\prime}}^{-}\right)\right]
\end{aligned}
$$

where the summation over the index $b=\{0, z\}$ and the limit $\Omega \rightarrow 0$ are assumed. We have also used the notations $\eta_{0}=$ $n_{\text {imp }}\left(V_{\uparrow}+V_{\downarrow}\right)^{2} / 4$ and $\eta_{z}=n_{\text {imp }}\left(V_{\uparrow}-V_{\downarrow}\right)^{2} / 4$. Using the same approximations as for the undressed part of the response function we obtain the intrinsic contribution as

$$
\chi_{\mathrm{in,a}}^{(1)-}=-\frac{e \alpha_{R} \Delta}{8 M} v_{0} \frac{\gamma_{\uparrow}+\gamma_{\downarrow}}{2\left(\gamma_{\uparrow} \gamma_{\downarrow}\right)^{\frac{1}{2}}} E_{a},
$$

while the corresponding extrinsic contribution is of second order in scattering rates and can be neglected. 
Thus, we obtain the FL and ADL torques in the limit $\gamma_{s} \ll$ $\alpha_{R} k_{F} \ll M$ to leading order in the SOI as

$$
\begin{gathered}
\boldsymbol{T}_{\mathrm{FL}}=\frac{e \alpha_{R} \nu_{0} \Delta}{M}\left[\frac{\varepsilon_{F}-M}{\gamma_{\downarrow}}-\frac{\varepsilon_{F}+M}{\gamma_{\uparrow}}\right] \boldsymbol{m} \times(\hat{z} \times \boldsymbol{E}), \\
\boldsymbol{T}_{\mathrm{ADL}}=\left[\frac{\gamma_{\uparrow}+\gamma_{\downarrow}}{2 \sqrt{\gamma_{\uparrow} \gamma_{\downarrow}}}-1\right] 2 e \alpha_{R} \nu_{0} \boldsymbol{m} \times(\boldsymbol{m} \times(\hat{z} \times \boldsymbol{E})) .
\end{gathered}
$$

These expressions provide the main result of this paper.

\section{CONCLUSIONS}

The SOT mechanism is based on the exchange of angular momentum between the crystal lattice and the local magnetization via spin-orbit coupling. Here, we found the FL and ADL SOTs microscopically, Eqs. (18) and (19). The FL SOT originates from the Fermi surface contribution of the response function, Eq. (8), while the ADL SOT is acquired contributions from the entire bands. Our main result, in Eq. (19), immediately shows that the intrinsic contribution to ADL SOT is completely canceled in the presence of spin-independent scattering $\gamma_{\uparrow}=\gamma_{\downarrow}$. That is, the intrinsic component of the ADL SOT, which originates from virtual interbranch transitions, is canceled by the vertex correction when weak spin-independent impurity scattering is taken into account. Our result, therefore, explicitly elucidates the interplay between intrinsic and extrinsic contributions to ADL SOT. This result resembles the suppression of both spin Hall conductivity in nonmagnetic metals and anomalous Hall conductivity in magnetic metals, in the presence of spinindependent disorder [44-48]. In these effects the cancellation is model dependent and occurs for parabolic band dispersion and linear-in-momentum SOI. We expect a similar scenario for intrinsic SOT.

The existence of a Rashba effect on the interface between an ultrathin ferromagnet and a heavy metal is the subject of intense discussion. Our results show that the amplitudes of the FL and ADL SOTs can be of the same order of magnitude, depending on the relative strengths of the SOI, spin-dependent scattering rates, and exchange interaction. Our results may qualitatively describe $\mathrm{Co} / \mathrm{Pt}$ interfaces, which are characterized by particularly large Rashba SOIs, of the magnitude of $1 \mathrm{eV} \AA$ [26-28,49]. Relating the strength of the Rashba coupling to the magnitude of the SOTs, however, would require ab initio modeling and additional experimental information. Which of our results apply to more general models and band structures will be the subject of future investigation.

\section{ACKNOWLEDGMENTS}

We acknowledge Hiroshi Kohno and Dmitry Yudin for useful discussions. The work was supported by Dutch Science Foundation NWO/FOM 13PR3118 and by EU Network FP7PEOPLE-2013-IRSES Grant No. 612624 "InterNoM". R.D. is a member of the D-ITP consortium, a program of The Netherlands Organisation for Scientific Research (NWO), which is funded by the Dutch Ministry of Education, Culture and Science (OCW).
[1] A. Manchon, Nat. Phys. 10, 340 (2014).

[2] T. Kuschel and G. Reiss, Nat. Nanotechnol. 10, 22 (2015).

[3] J. Sinova and I. Žutić, Nat. Mater. 11, 368 (2012).

[4] I. Žutić, J. Fabian, and S. Das Sarma, Rev. Mod. Phys. 76, 323 (2004).

[5] J. Sinova, S. O. Valenzuela, J. Wunderlich, C. H. Back, and T. Jungwirth, Phys. Rev. Mod. (to be published), arXiv:1411.3249.

[6] N. Nagaosa, J. Sinova, S. Onoda, A. H. MacDonald, and N. P. Ong, Rev. Mod. Phys. 82, 1539 (2010).

[7] V. M. Edelstein, Solid State Commun. 73, 233 (1990).

[8] J.-I. Inoue, G. E. W. Bauer, and L. W. Molenkamp, Phys. Rev. B 67, 033104 (2003).

[9] P. Gambardella and I. M. Miron, Phil. Trans. R. Soc. A 369, 3175 (2011).

[10] A. Brataas and K. M. D. Hals, Nat. Nanotechnol. 9, 86 (2014).

[11] K. M. D. Hals and A. Brataas, Phys. Rev. B 88, 085423 (2013).

[12] C. Ciccarelli, K. M. D. Hals, A. Irvine, V. Novak, Y. Tserkovnyak, H. Kurebayashi, A. Brataas, and A. Ferguson, Nat. Nanotechnol. 10, 50 (2015).

[13] N. Locatelli, V. Cros, and J. Grollier, Nat. Mater. 13, 11 (2013).

[14] A. Brataas, A. D. Kent, and H. Ohno, Nat. Mater. 11, 372 (2012).

[15] D. C. Ralph and M. D. Stiles, J. Magn. Magn. Mater. 320, 1190 (2008).

[16] B. A. Bernevig and O. Vafek, Phys. Rev. B 72, 033203 (2005).

[17] A. Manchon and S. Zhang, Phys. Rev. B 78, 212405 (2008); 79, 094422 (2009).
[18] A. Matos-Abiague and R. L. Rodríguez-Suárez, Phys. Rev. B 80, 094424 (2009).

[19] I. Garate and A. H. MacDonald, Phys. Rev. B 80, 134403 (2009).

[20] X. Wang and A. Manchon, Phys. Rev. Lett. 108, 117201 (2012).

[21] K.-W. Kim, S.-M. Seo, J. Ryu, K.-J. Lee, and H.-W. Lee, Phys. Rev. B 85, 180404(R) (2012).

[22] D. A. Pesin and A. H. MacDonald, Phys. Rev. B 86, 014416 (2012).

[23] A. Chernyshov, M. Overby, X. Liu, J. K. Furdyna, Y. LyandaGeller, and L. P. Rokhinson, Nat. Phys. 5, 656 (2009).

[24] I. M. Miron, T. Moore, H. Szambolics, L. D. Buda-Prejbeanu, S. Auffret, B. Rodmacq, S. Pizzini, J. Vogel, M. Bonfim, A. Schuhl, and G. Gaudin, Nat. Mater. 10, 419 (2011).

[25] A. V. Khvalkovskiy, V. Cros, D. Apalkov, V. Nikitin, M. Krounbi, K. A. Zvezdin, A. Anane, J. Grollier, and A. Fert, Phys. Rev. B 87, 020402(R) (2013).

[26] I. M. Miron, G. Gaudin, S. Auffret, B. Rodmacq, A. Schuhl, S. Pizzini, J. Vogel, and P. Gambardella, Nat. Mater. 9, 230 (2010).

[27] I. M. Miron, K. Garello, G. Gaudin, P.-J. Zermatten, M. V. Costache, S. Auffret, S. Bandiera, B. Rodmacq, A. Schuhl, and P. Gambardella, Nature 476, 189 (2011).

[28] K. Garello, I. M. Miron, C. O. Avci, F. Freimuth, Y. Mokrousov, S. Blügel, S. Auffret, O. Boulle, G. Gaudin, and P. Gambardella, Nat. Nanotechnol. 8, 587 (2013).

[29] K. Garello, C. O. Avci, I. M. Miron, M. Baumgartner, A. Ghosh, S. Auffret, O. Boulle, G. Gaudin, and P. Gambardella, Appl. Phys. Lett. 105, 212402 (2014). 
[30] J. Železný, H. Gao, K. Výborný, J. Zemen, J. Mašek, A. Manchon, J. Wunderlich, J. Sinova, and T. Jungwirth, Phys. Rev. Lett. 113, 157201 (2014).

[31] P. Wadley, B. Howells, J. Železný, C. Andrews, V. Hills, R. P. Campion, V. Novák, F. Freimuth, Y. Mokrousov, A. W. Rushforth, K. W. Edmonds, B. L. Gallagher, and T. Jungwirth, arXiv:1503.03765.

[32] J. Linder, Phys. Rev. B 87, 054434 (2013).

[33] E. van der Bijl and R. A. Duine, Phys. Rev. B 86, 094406 (2012).

[34] L. Liu, C.-F. Pai, Y. Li, H. W. Tseng, D. C. Ralph, and R. A. Buhrman, Science 336, 555 (2012).

[35] J. Kim, J. Sinha, M. Hayashi, M. Yamanouchi, S. Fukami, T. Suzuki, S. Mitani, and H. Ohno, Nat. Mater. 12, 240 (2013).

[36] X. Fan, H. Celik, J. Wu, C. Ni, K.-J. Lee, V. O. Lorenz, and J. Q. Xiao, Nat. Commun. 5, 3042 (2014).

[37] G. Yu, P. Upadhyaya, Y. Fan, J. G. Alzate, W. Jiang, K. L. Wong, S. Takei, S. A. Bender, L.-T. Chang, Y. Jiang, M. Lang, J. Tang, Y. Wang, Y. Tserkovnyak, P. K. Amiri, and K. L. Wang, Nat. Nanotechnol. 9, 548 (2014); I. M. Miron, ibid. 9, 502 (2014).

[38] P. M. Haney, H.-W. Lee, K.-J. Lee, A. Manchon, and M. D. Stiles, Phys. Rev. B 87, 174411 (2013).

[39] H. Kurebayashi, J. Sinova, D. Fang, A. C. Irvine, T. D. Skinner, J. Wunderlich, V. Novák, R. P. Campion, B. L. Gallagher,
E. K. Vehstedt, L. P. Zârbo, K. Výborný, A. J. Ferguson, and T. Jungwirthn, Nat. Nanotechnol. 9, 211 (2014).

[40] H. Li, H. Gao, L. P. Zârbo, K. Výborný, X. Wang, I. Garate, F. Doğan, A. Čejchan, J. Sinova, T. Jungwirth, and A. Manchon, Phys. Rev. B 91, 134402 (2015).

[41] F. Freimuth, S. Blügel, and Y. Mokrousov, Phys. Rev. B 90, 174423 (2014).

[42] R. A. Duine, A. S. Núñez, J. Sinova, and A. H. MacDonald, Phys. Rev. B 75, 214420 (2007).

[43] D. Bercioux and P. Lucignano, arXiv:1502.00570.

[44] T. Kato, Y. Ishikawa, H. Itoh, and J.-I. Inoue, New J. Phys. 9, 350 (2007).

[45] C. P. Moca and D. C. Marinescu, New J. Phys. 9, 343 (2007).

[46] T. S. Nunner, N. A. Sinitsyn, M. F. Borunda, V. K. Dugaev, A. A. Kovalev, A. Abanov, C. Timm, T. Jungwirth, J.-I. Inoue, A. H. MacDonald, and J. Sinova, Phys. Rev. B 76, 235312 (2007).

[47] J.-I. Inoue, T. Kato, Y. Ishikawa, H. Itoh, G. E. W. Bauer, and L. W. Molenkamp, Phys. Rev. Lett. 97, 046604 (2006).

[48] A. Sakai, Ph.D. thesis, Osake University (2014); A. Sakai and H. Kohno (unpublished).

[49] J.-H. Park, C. H. Kim, H.-W. Lee, and J. H. Han, Phys. Rev. B 87, 041301(R) (2013). 